\title{
MYO-ELECTRIC CONTROL OF A HAND PROSTHESIS
}

\author{
J. B. Knowles, B. L. Stevens and L. Howe, Manchester, England
}

Over the past three years at the Manchester College of Science and Technology there has been research into the myo-electric control of a hand prosthesis. At present three engineers are engaged on the work, and the necessary medical collaboration is obtained from staff of the Liverpool and Manchester Regional Hospital Boards. The ultimate object of the research is the production of a fully articulated hand capable of several natural finger movements and an independent wrist movement.

A rudimentary hand prosthesis capable of a "chuck" grip to a three-point closure position was developed in 1962. A schematic diagram of the electrical mechanisms involved is shown in Figure 1, and its mode of operation is as follows. Muscular activity beneath a skin surface electrode induces small impulsive changes in the electrical potential of that electrode with respect to the common electrode $\mathrm{E}$. These electrical pulses are amplified by means of the amplifiers $A_{1}$ and $A_{2}$ to a workable voltage level. After rectification and smoothing each electrical signal has an amplitude approximately proportional to the tension developed in the respective muscle. Electronic subtaction of these signals and subsequent power amplification provides a controlling signal which drives the piston of the stepping motor appropriately forwards or backwards, thereby producing the desired movement of flexion or

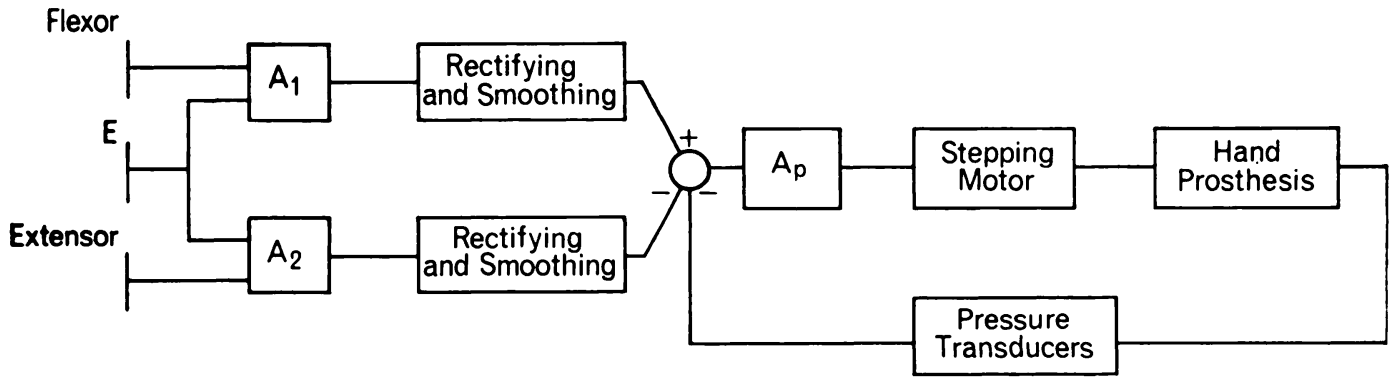

FIG. 1

Schematic layout of the electrical circuit. $A_{1} A_{2}=$ Amplifiers; $A p=$ Power amplifier; $E=$ common electrode.

extension in the prosthesis. When the digits of the prosthetic hand close on an object, an electrical signal proportional to the grip pressure is derived from pressure transducers. This " sensory" signal is subtracted from the dominant flexion signal, and the motor drive power is correspondingly reduced. Equilibrium is established when the signals giving flexion and grip pressure are of the same magnitude. With this type of control system any lightly gripping posture may be maintained with slight effort on the part of the wearer. Furthermore, a heavy grip is only sustained by considerable effort on the part of the wearer. In these respects, the prosthesis closely resembles the natural operation of the hand.

The fully articulated prosthetic hand was actually constructed of aluminium and its digits were of rectangular cross-section with crudely pinned mortise and tenon joints. Flexion was achieved by means of bowdon cables attached to the piston of the stepping motor and extension was obtained by means of return springs. Springs were incorporated in the "flexor" tendons to facilitate the handling of irregular objects. The " sensory pressure" feedback described above was obtained through the use of carbon-bonded rubber on all palmar surfaces of the prosthesis. By measuring the change of resistance induced in this material by external compression, an electrical signal was derived proportional to the pressure on the held object. An illustration of the prosthesis holding an object is shown in Figure 2. It should be emphasised that the device as at present constructed is crude and unfinished. 
Having made a fundamental engineering appraisal of the problem of the hand prosthesis with the aid of the prototype described above, a systematic approach towards the production of a fully articulated hand capable of natural finger movements and independent wrist movement was started. Over the past year attention has been directed towards the development of a transistorised myo-electric amplifier suitable for microminiaturisation. Transistorised biological amplifiers already in existence are not amenable to microminiaturisation because of the large transformers they contain. Using normal transistors, an amplifier has been made in which the input transformer has been eliminated. A photograph of the device in a development stage is shown in Figure 3, and the pertinent details of its performance are as follows: 3dB Bandwidth: 10-7,000 c/s, Common Mode Resistance 1.5 M 2 . Peak-to-peak output voltage swing \pm 3 volts. Peak-to-peak noise referred to input for a short circuited input $3.5 \mu$ volts.

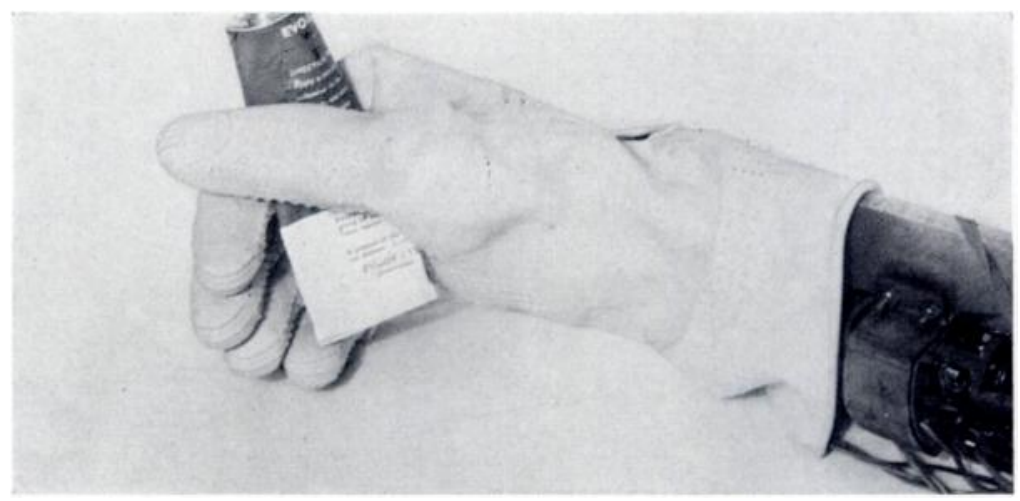

Fig. 2

The artificial hand holding an object.

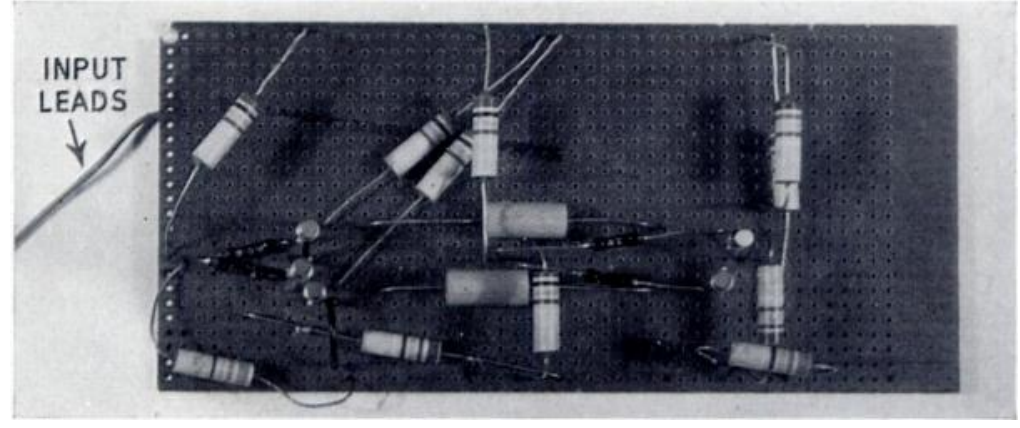

Fig. 3

The device in the development stage.

Because a microminiaturised amplifier can be made to be virtually integral with its electrodes, the amount of spurious $50 \mathrm{c} / \mathrm{s}$ interference which invariably contaminates the amplifier output can be made very small. Discussions with orthopaedic surgeons and physiologists have led us to believe that such an amplifier would be of value in clinical electromyography. With this application, portability and the elimination of the costly electrically screened room are the significant factors. In fact, the surgeon could carry the amplifier complete with power supplies in his top pocket, and with a portable lightweight oscilloscope recordings could be made at any part of the hospital.

In the immediate future it is hoped to develop for the electric motor simple controls possessing greater power economy. Furthermore, an investigation is planned into the effect of processing the muscle potentials so as to obtain a more accurate estimate of muscle tension. 